\title{
Design of PZT Power Supply for Micro-nano Positioning Platform Based on DSP
}

\author{
Hao Lina ${ }^{\mathrm{a}^{*}}$, Zhang Xinchao ${ }^{\mathrm{b}}$, Cao Ruimin ${ }^{\mathrm{c}}$ \\ School of Mechanical Engineering \& Automation, Northeastern University, Shenyang, China. \\ aEEmail: haolina@me.neu.edu.cn(corresponding author), ${ }^{\mathrm{a} E m a i l:}$ ZHANGXIN_CHAO@126.com , \\ 'Email: xiaojiacaoruimin@126.com,
}

Keywords: Micro-nano positioning platform; PZT; DSP; PZT power supply

Abstract. PZT is the key actuator to high precision micro-nano positioning platform. Based on high large space density precision micro-nano positioning platform, we use DSP and four roads 16-bit DAC, high order low pass filter, the voltage amplifier circuit, power amplifier circuit, high voltage stabilizing circuit and phase compensating circuit to design PZT power supply. And also we use Multisim12 to simulate it. Through the simulation and experimental validation, the PZT power supply has continuous adjustable dc voltage $0-150 \mathrm{~V}$, and the ripple is under $8 \mathrm{mV}$. Then it has fast dynamic response, good stability, strongly driving ability and so on. So the PZT power supply can completely satisfy the micro-nano positioning platform.

\section{Introduction:}

PZT is a new kind of micro displacement actuator which is developed in recent years. It has small size, large thrust, no friction, accuracy, high resolution, fast frequency response characteristics and so on, and it is used without noise, no thermal effect [1]. So it is an ideal micro displacement actuator. The PZT power supply has a great influence on the performance of the PZT. But now the types of PZT power supply on market are few. And also the price is high. The dynamic characteristic of a single channel is not ideal. And they have few channels, big volume. So they are not suitable for the application in high precision micro-nano positioning platform. So it is necessary to design a kind of PZT power supply for micro-nano positioning platform. And it must satisfy the designed high precision positioning platform and have more channels, low cost,small volume, high precision and good dynamic characteristics.

\section{The Control System of Micro-nano Positioning Platform}

Six parts consist of the control system of the high precision positioning platform. They are PC, the center of the measurement and control module based on DSP and FPGA, the linear amplifier module, the communication module of PC, the displacement signal generation module, and displacement signal receiving module. The platform system is shown in Fig 1.

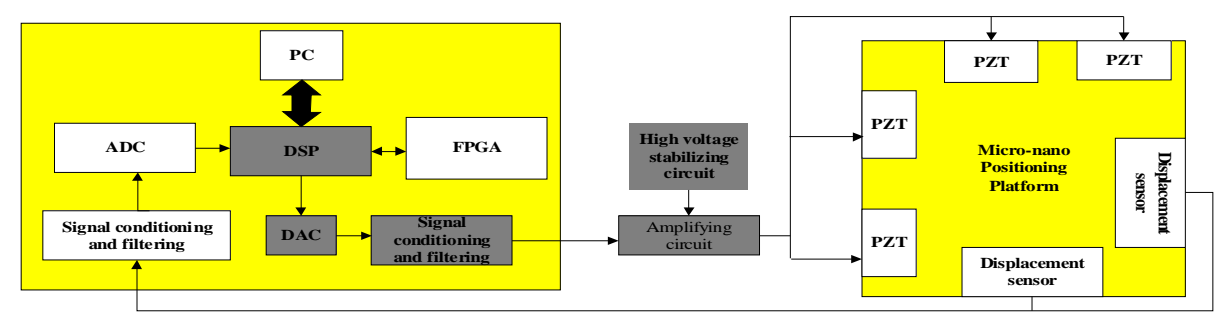

Fig 1 Control system of micro-nano positioning platform 


\section{The Composition of PZT Power Supply}

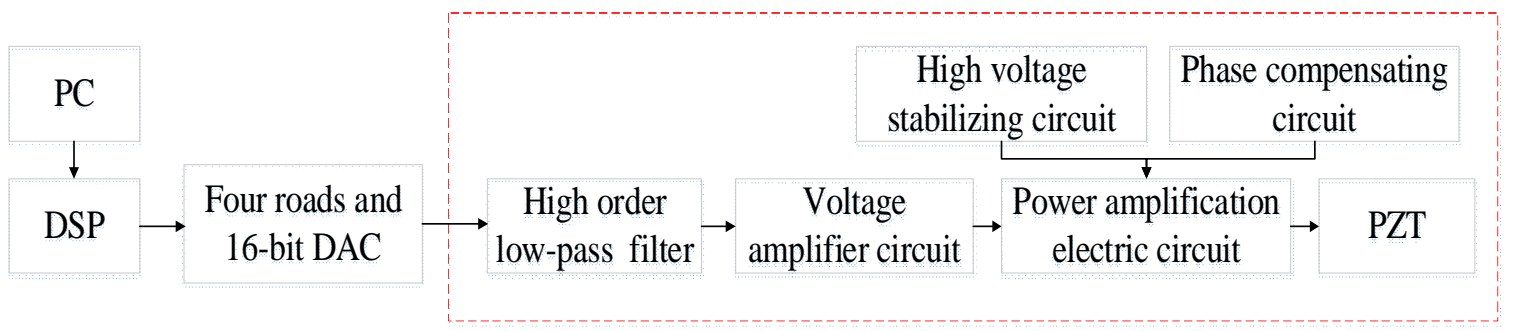

Fig2 Principle diagram of PZT Power Supply

According to the characteristics of PZT, the driving power supply requires continuous adjustable within a certain range, output stability, small ripple, high resolution [2]. The component of New PZT power supply is shown in figure 2. It is mainly composed of four roads 16-bit high-precision DAC, high order low pass filter, the voltage amplifier circuit, power amplifier circuit, high voltage stabilizing circuit and phase compensating circuit. Because the PZT power supply has four channels, the red part has four, respectively connected to the pins of the four roads 16-bit DAC.

\section{Design of the PZT Power Supply}

\section{The Design of Signal Generator}

The signal generator is mainly formed by the connection of computer, DSP and DAC8534. DSP chip uses TM320F2812 which is a 32-bit fixed-point DSP form TI [3]. This article adopts the high-speed clock SPI interface of DSP and the DAC chip connection, whose biggest characteristic is the high speed of data transmission.

\section{Linear Amplifier Circuit}

In this paper, PZT power supply uses dc amplification principle [4]. Continuous adjustable dc voltage $0-150 \mathrm{~V}$ is obtained by high voltage linear amplifier circuit to drive the PZT. This article uses the PA85 and OP07 series. The amplifier circuit is shown in figure 3.

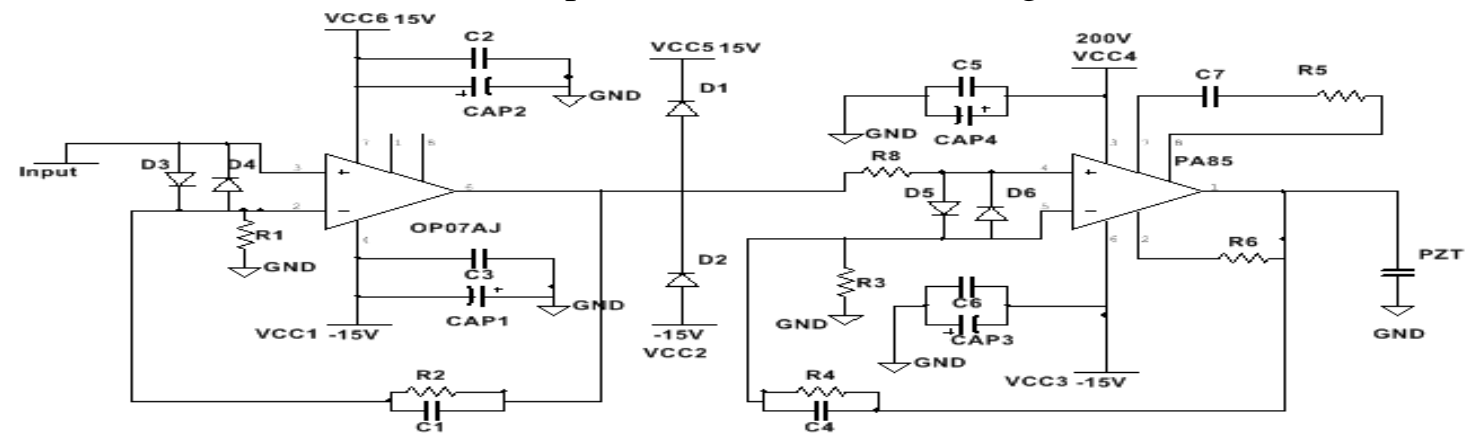

Fig3 Linear amplifier circuit

Select the integrated operational amplifier:

(1) The largest output current $\mathrm{I}_{\mathrm{lim}}$ : Maximum output current and current limiting resistance R6 approximate relation: $I_{\lim }=0.7 / \mathrm{R} 6$ [4]. This design chooses $3.5 \Omega$ current-limiting resistor, and maximum output current is $200 \mathrm{~mA}$.

(2) Calculation of maximum frequency of PZT: The highest frequency of the sinusoidal current to the PZT is $f_{\max }$. The peak - peak input voltage value is $\mathrm{V}_{p p}$ and input max current is $I_{p}$. The equivalent capacitance is $C_{l}$. They are based on the relationship: 


$$
f_{\text {max }}=\frac{I_{p}}{\pi \times V_{p p} \times C_{l}}
$$

$V_{p p}=150 \mathrm{~V}, C_{l}=1.5 \mu \mathrm{F}, I_{p}=200 \mathrm{~mA}$. So we can get $\mathrm{f}_{\max }=282.9 \mathrm{~Hz}$ [5]. Then the highest frequency of the output of the sine voltage is $282.9 \mathrm{~Hz}$. The PA85 is a high pressure, large bandwidth MOSFET operational amplifier. The output current reaches $200 \mathrm{~mA}$. When single side supplied, the output voltage can be up to $440 \mathrm{~V}$ [6]. So it can meet the requirements.

\section{The Stability Analysis of Linear Amplifier Circuit}

The input offset voltage is decided by the smaller input offset voltage OP07. The maximum offset voltage of OP07 is $75 \mu \mathrm{V}$. So the maximum offset voltage of power supply is $75 \mu \mathrm{V} \times 30=2.25$ $\mathrm{mV}<8 \mathrm{mV}$, and it meets the requirements of input features.

\section{The Allocation of Magnification}

The input voltage of composite amplifier is $0-5 \mathrm{~V}$. The output voltage is $0-150 \mathrm{~V}$. So it is concluded that magnification is 30 . According to the distribution of the magnification, R1,R2 and $\mathrm{R} 3$ are $3 \mathrm{k} \Omega$, and $\mathrm{R} 4$ is $42 \mathrm{k} \Omega$. So the total magnification of the two levels for $A$ is as follows:

$$
A=\left(1+\frac{R 2}{R 1}\right) \times\left(1+\frac{R 3}{R 4}\right)
$$

PA85 closed-loop magnification is 15 , thus we calculate that the gain is 14. According to APEX PA85 data provided by the company, the gain is relatively close to 20[7].Thus we determine the phase compensation resistor and capacitor value is as follows: $\mathrm{R} 5=330 \Omega \mathrm{C} 8=10 \mathrm{pF}$. Through the instruction, the circuit can compensate the phase difference very well.

\section{Simulation and Experiment of the PZT Power Supply}

\section{Dynamic Characteristics Simulation of Linear Amplifier Module}

Multisim12 is circuit simulation analysis software launched by NI. It provides a good interface and fast operation, and has strong analysis function. So in this paper, we use the software to simulate the dynamic characteristics of linear amplifier module. We test peak-peak value is $5 \mathrm{~V}$, frequency is $30 \mathrm{~Hz}$ and peak-peak value is $1.5 \mathrm{~V}$, frequency is $280 \mathrm{~Hz}$ sine wave,triangle wave and step signal. The input and output are shown in figure 4.1, 4.2, 4.3, 4.4, 4.5 and 4.6.

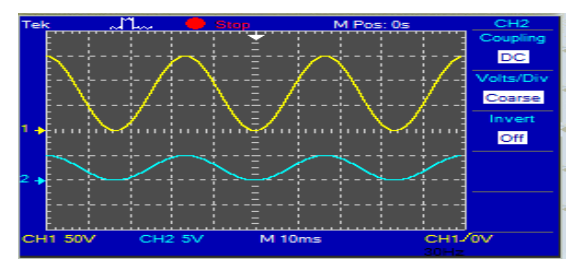

Fig4.1 5V/30Hz

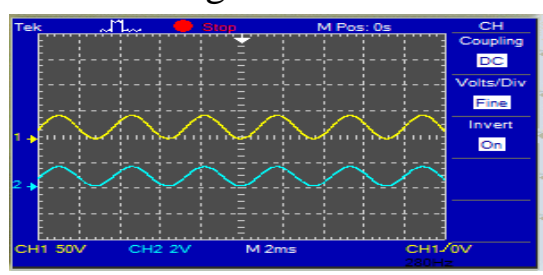

Fig4.4 1.5V/280Hz

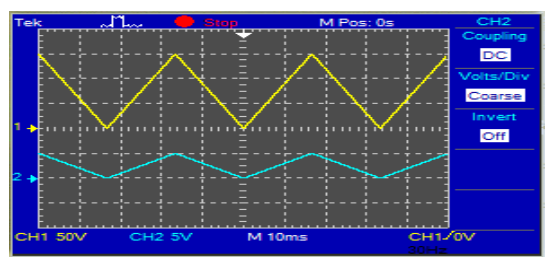

Fig4.15V/30Hz

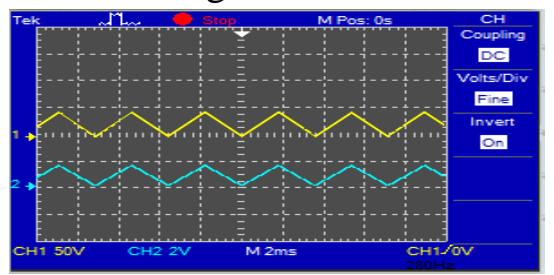

Fig4.5 1.5V/280Hz

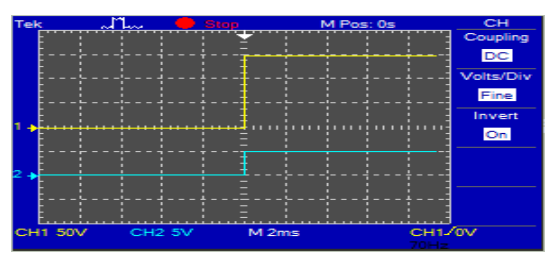

Fig4.3 15V step signal

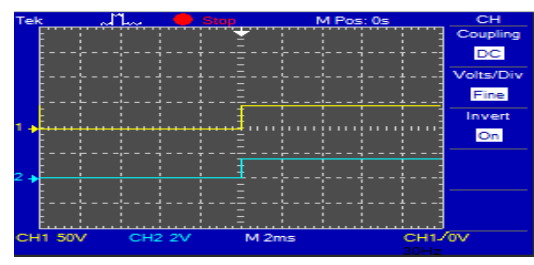

Fig4.6 1.5V step signal

The dynamic characteristics of the simulation shows that the PZT power supply performance is good, and the circuit has a good stability, fast response and load capacity. 


\section{Static Characteristic Simulation of High Voltage Linear Amplification Module}

We simulated the static characteristic of the linear amplifier circuit. The data is shown in Table1:

Table1 the static characteristic simulation data of high voltage linear amplification module

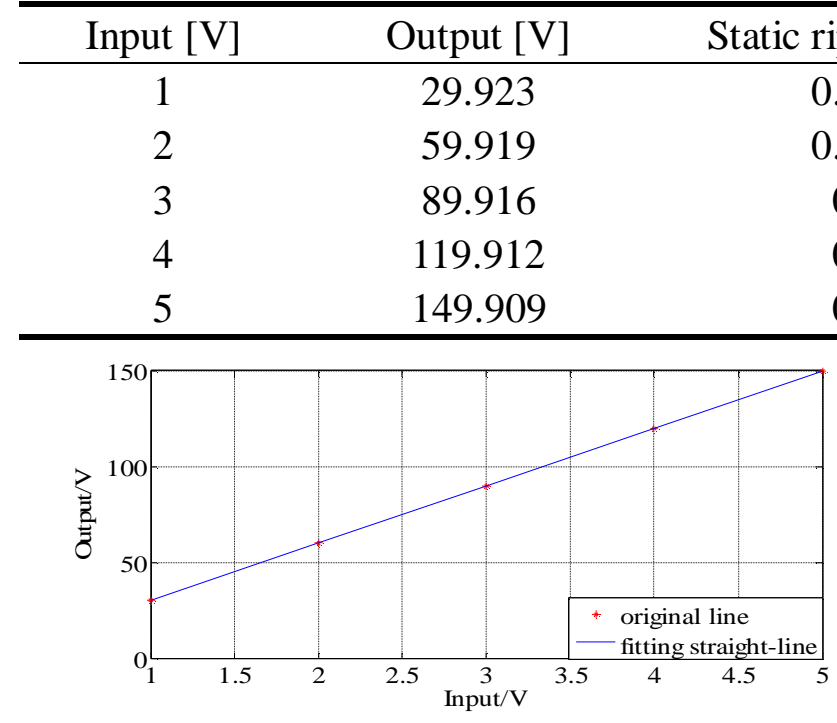

Fig5 Fitting straight line

$\begin{array}{cc}0.075 & 199.683 \\ 0.076 & 199.705 \\ 0.7 & 199.729 \\ 0.4 & 199.752 \\ 0.9 & 199.775\end{array}$

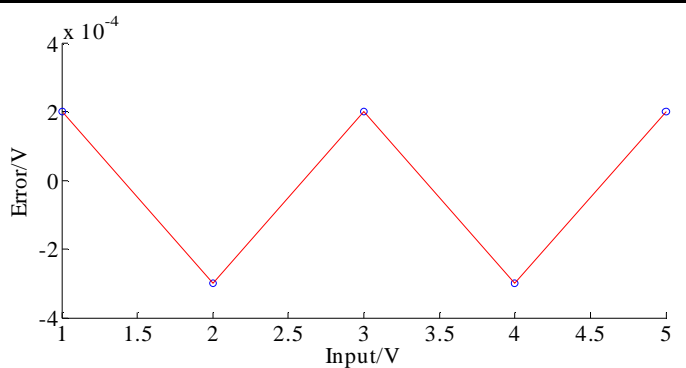

Fig6 Error straight lines

The simulation shows that maximum error is in $29.923 \mathrm{~V}$, the straightness test error is 0.0002 , and the linear correlation coefficient is nearly 1.0.Therefore, the linearity of linear amplification circuit is very good. Through the simulation test, the circuit can achieve the maximum voltage 150V, and has low ripple. So it meets the requirement.

\section{Experiment of PZT Power Supply}

Experimental apparatus is shown in the figure 7:

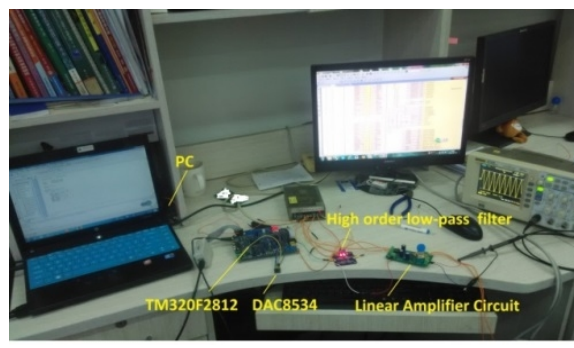

Fig 7 The experimental device

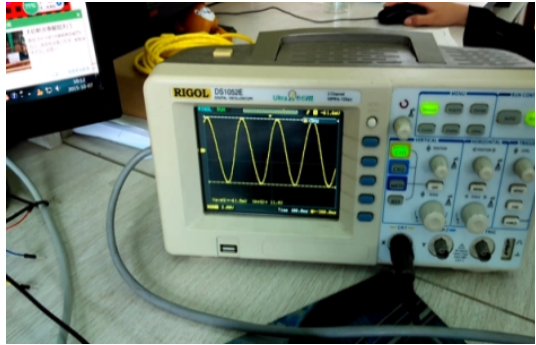

Fig 8 The output sine wave

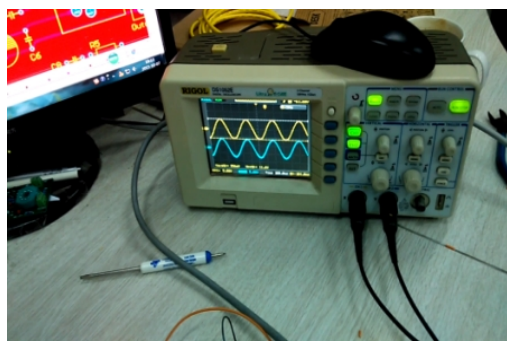

Fig 9 I/O comparison

Because the current experiment equipment is unable to test the performance of full voltage, we only tested 0-1.5 V input performance as a representative. When the peak-peak voltage of sine wave is $1.5 \mathrm{~V}$,the frequency is $280 \mathrm{~Hz}$, the output sine wave is shown in Fig 8, and the comparison of input and output is show in Fig 9. We tested static characteristic of the linear amplifier circuit, the data is shown in Table2: 
Table2 the static characteristic experiment data of linear amplification module

\begin{tabular}{cccc}
\hline Input [V] & Output [V] & Static ripple [mV] & Maximum working current [mA] \\
\hline 0.2 & 6.46 & 4.07 & 192 \\
0.4 & 11.89 & 4.11 & 192 \\
0.6 & 18.42 & 4.39 & 192 \\
0.8 & 24.24 & 5.37 & 192 \\
1.0 & 30.55 & 3.93 & 192 \\
1.2 & 35.75 & 4.03 & 192 \\
1.5 & 45.31 & 3.76 & 192 \\
\hline
\end{tabular}

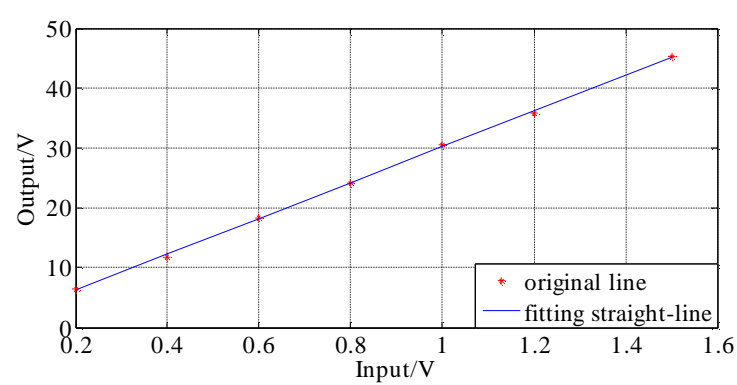

Fig10 Fitting straight line

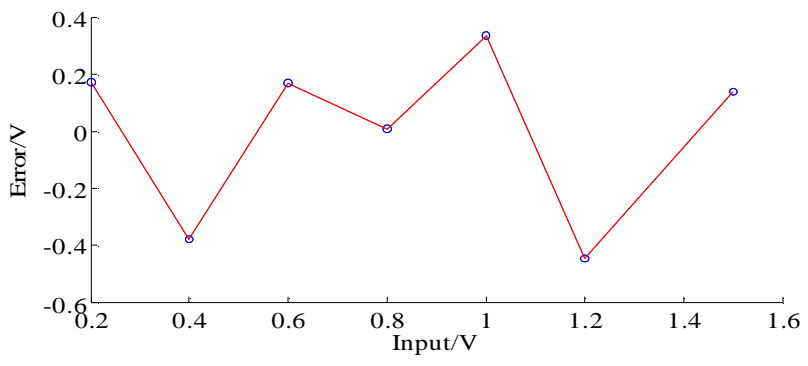

Fig11 Error straight lines

The experiment shows that output waveform is very stable, and it almost has no noise wave. And also the maximum error is in $30.55 \mathrm{~V}$, the straightness test error is $0.33 \mathrm{~V}$, and the linear correlation coefficient is 0.9998 . Therefore, the linearity of linear amplification circuit is very good. The ripple of PZT power supply is from 3 to $6 \mathrm{mV}$. So the steady state performance is good, and it can satisfy the high precision positioning platform to use. And also we give the power step signal, the corresponding up and down response time is less than $0.3 \mathrm{~ms}$. This suggests that it has good dynamic performance.

\section{Conclusion}

In this paper the PZT power supply based on DSP has high frequency resolution and real-time tracking. With high voltage amplifier and low-voltage op-amp complex structure, we realize high precision driving voltage control. At the same time it has reasonable phase compensation circuit and protection circuit. And it has a high stability and load capacity, and it completely can be applied to the designed high precision micro-nano positioning platform. In addition because of its high cost performance, simple structure, it has the very high practical value.

\section{Acknowledgement}

This work was sponsored by Science and Technology Plan Project of Shenyang (No.F13-316-1-74), Liaoning Bai Qian Wan Talents Program (No.2013921069) and the University Innovation Team of Liaoning Province (LT2014006).

\section{References}

[1] LIU Bo ,GUO Jian-ying ,SUN Yong-quan .Modeling and control for PZT microdisplacement Actuator[J].0ptics and Precision Engineering, 2013,21(6):1503-1508. 
[2] HE Bin, CHEN Hua , XIE Hongxia. An Piezoelectric Ceramic Driving Power Implemented by Digital Potentiometer Regulation [J]. Piezoelectrics \& Acoustooptics, 2012, 34(2): 253-256.

[3] Zhang Lei. Research on High-pressure Piezoelectric Ceramic Driving Power[D]. Harbin Institute of Technology.2014.

[4] WANG Hui, GONG Chi-kun,WANG Hong-feng .New piezoelectric ceramics drive power based on PA 95[J]. MICROCOMPUTER INFORMATION,2011,27(12):58-59

[5] Hu Jun, Luo Yunzhi, Tang Gongfu .Piezoelectric Ceramic Driving Power Supply Design for Beam Director [J]. Ordnance Industry Automation,2015, 34(4):43-45.

[6] Jie Chen. Research on Piezoelectric Ceramic Drive Power and Applications[D]. Nanjing University of Aeronautics and Astronautics. 2012.

[7] Li F. A New Power Supply for Piezoelectric Ceramic Based on PA85 [J]. Piezoelectrics \& Acoustooptics, 2005, 27(4): 392-394. 